$\Phi=5$

\title{
Assessment of the factors contributing to non- adherence of anti- retroviral therapy (ART) treatment among male patients at Katutura Health Centre, Khomas region
}

\author{
NiilongaElina N*, Nghitanwa Emma Maano \\ Faulty health Sciences, School of Nursing, University of Namibia, Windhoek, Namibia \\ *Corresponding author E-mail:enghitanwa@unam.na
}

\begin{abstract}
The purpose of this study was to assess and explore the contributing factors of non- adherence among male patients on Antiretroviral therapy (ART) treatment at Katutura Health Centre, Khomas Region. The objectives of this study were to determine and describe the factors contributing to non-adherence of ART treatment among male patients at Katutura Health Centre .Data were collected using the structured questionnaires.

A purposive sampling technique was used. The collected data were entered in data sets and analyzed using Statistical (SPSS) version 23.0 software to describe the phenomena and to assess the magnitude of ART non-adherence among male patients.

The study concluded that non-adherence of ART treatment among male patients in Katutura Health Centre, Khomas region is caused by different factors, such as, lack of food among patients on ART treatment, lack of transportation to the health facility for treatment and alcohol abuse. Therefore practical preventative interventions such as community education program should be developed and implemented to improve and ensure ART adherence.
\end{abstract}

Keywords: Antiretroviral Therapy; Factors; Male; Non-Adherence; Patients.

\section{Introduction}

Human Immunodeficiency Virus (HIV) attacks the body's immune system, especially the CD4 cells (T cells), which help the immune system fight off infections. According to the World Health Organization (WHO) (2006) currently there is no cure for HIV, but with proper treatment and antiretroviral therapy (ART) adherence, HIV infected person's quality of life could be improved.

Adherence to ART is the crucial issue in HIV/AIDS therapeutics in order to build up the immune system, prevention of drug resistance and achieve viral suppression (Makua, 2015). Furthermore, ART adherence of more than $95 \%$ is required for a successful and effective HIV management. Adherence, refers to the taking correct prescribed medication, correct doses at correct prescribed time in order to suppress HIV and to prevent drug resistance (Bauleth et al., 2016).To determine adherence, different methods are being used such as self-report, interview, pharmacy record, pill count and viral load monitoring.

Several studies has reported factors related to non-adherence of ART treatment such as lack of family support, depression and confusion (Tjituka et al., 2013 ), stigma and discrimination, spiritual beliefs ,forget to take medication(Bauleth, 2016),side effects of medication (Demeke and Chanie , 2014) and workplace factors (Reda\&Biadgilign, 2012). Furthermore, socio-economic factors such as alcohol abuse and transport costs, health related factors such as long waiting times and health professionals' negative attitudes were also reported as factors contributing to non-adherence of ART treatment (Bauleth, 2013).
Namibia is among the countries with highest HIV prevalence in the world, with $16.9 \%$ of the adult population reported as living with HIV in 2014 (Ministry of Health and Social Service, 2014) .The Ministry of Health and Social Service (MoHSS) of Namibia has rolled up HIV treatment in the public health facilities by distributing ART treatment free of charge to people infected by HIV since 2003 (MoHSS, 2014). The introduction of ART services resulted in to the reduction of HIV/AIDS related morbidity and mortality among HIV infected individuals globally (Kip et al., 2009; UNAIDS, 2006).

Katutura health center in the only health center in Windhoek, the capital city of Namibia. Windhoek has an estimated HIV/AIDS prevalence rate of about $15 \%$, with about 3.5 percent new infections per day (MoHSS, 2014). According to the MoHSS (2012/2013) annual report, since the beginning of the ART treatment program, active participation of men remains very poor, with the rate of non-adherence to ART treatment reported to be higher among male than female patients. In Katutura Health Centre, male patients initiated on ART in 2014 were 271 and 183 male patients were non adherent to ART treatment, while in 2015, 242 male patients were initiated in the ART and 173 were non adherent (MoHSS, Katutura Health Center report, 2016). This represented $68 \%$ non-adherence in 2014, and $71 \%$ in 2015 respectively. Moreover, poor adherence is one of the keys obstacles to successful ART (Bauleth et al., 2016).However, there is a paucity of published data on ART non adherence among male patients in Namibia. 


\section{Purpose}

The purpose of the study was to assess and explore the contributing factors to non-adherence among male patients on ART treatment at Katutura Health Centre, Khomas Region, Namibia.

\section{Objectives}

The objectives of the study were to:

- Describe the socio-demographic characteristics of participants

- Determine the factors contributing to non-adherence of ART treatment among male patients at Katutura Health Centre, Khomas region.

\section{Methodology}

A cross sectional, quantitative, descriptive study design was used to determine the factors contributing to non-adherence among male patients on ART. The target population was all male patients who did not adhere to the ART treatment during the period of 6 months from January 2016 to June 2016.The inclusion criteria were: male patients aged 18 years and above identified as nonadherent to ART treatment who were willing to participate in the study. Male patients who were non adherent to ART treatment less than 18 years old were excluded, since they cannot make decisions to take part in the study, so they would need parental consent. According to the MoHSS, Katutura Health Center statistic (2016)79 male patients who met inclusion criteria did not adhere to ART treatment during January 2016 to June 2016. Census sampling was employed to recruit all 79 non adherent male patients. However, only 66 male patients were available and agreed to participate in the study, resulting in the response rate of $84 \%$.

The registered nurse in charge of Katutura Health Center assisted in the recruitment of participants by providing the list of names, address and mobile numbers for ART non adherent male patients during the selected time frame as a sampling frame. All patients in the sampling frame were called telephonically and the study purpose was explained then they were asked whether they would like to participate in the study. Furthermore, participants were called telephonically to arrange the date, time and the venue of the data collection.

A structured questionnaire was developed by the researchers and verified by the competent professional in the field. The interviews were conducted in a private place of their choice and it was mostly in their homes or at Katutura Health center in a private office. Data was collected for a period of two months, from August 2016 to September 2016.Data entries were done in the SPSS software data set and data was analysed using SPSS software version 23.Descriptive statistics was used to analyse the sociodemographic characteristics of the participants as well as the factors contributing to the non- adherence to ART treatment among male patients.

\section{Ethical issues}

Permission to conduct research was obtained from the University of Namibia, School of Nursing research ethical committee and the Ministry of Health and Social Services. The purpose of the study and the right to participate or to withdraw from the study was explained to the participants. Participants were voluntary participated by signing the informed consent before commenced with the study. This was done to ensure the principle of respect, justice, autonomy and beneficence. Confidentiality and anonymity were maintained by not writing names of the participants on the questionnaires so that participant's name could not be linked with answers.

\section{Findings}

\subsection{Socio-demographic data}

There were 66 participants who were interviewed. The participants were all males with the mean age of 36.78. As displayed in table 1 the majority of the participants were within the age range of 25 to 34 while few were aged 55 years and above. Most of the participants were single $(69.7 \%)$ followed by married $(22.8 \%)$. Moreover, most participants $(60.6 \%)$ had secondary education and above half of participants $(57.6 \%)$ were unemployed.

Table 1: Socio-Demographic Data of Participants

\begin{tabular}{|c|c|c|}
\hline Variable & Frequency (n) & Percentage $\%$ \\
\hline \multicolumn{3}{|l|}{ Age category } \\
\hline $18-24$ & 11 & 16.6 \\
\hline $25-34$ & 21 & 32.0 \\
\hline $35-44$ & 15 & 22.7 \\
\hline $45-54$ & 14 & 21.2 \\
\hline 55 and above & 5 & 7.5 \\
\hline \multicolumn{3}{|l|}{ Marital status } \\
\hline Single & 46 & 69.7 \\
\hline Married & 15 & 22.8 \\
\hline Divorced & 2 & 3.0 \\
\hline Widow/er & 3 & 4.5 \\
\hline \multicolumn{3}{|c|}{ Education level } \\
\hline Primary & 8 & 21.0 \\
\hline Secondary & 40 & 60.6 \\
\hline Tertiary & 18 & 27.0 \\
\hline \multicolumn{3}{|c|}{ Employment status } \\
\hline Employed & 28 & 42.4 \\
\hline Unemployed & 38 & 57.6 \\
\hline
\end{tabular}

\subsection{Factors relating to non-adherence to ART treat- ment among male patients}

Participants indicated several factors which prevent them to adhere to ART treatment such as social factors, economic factors, employment factors, treatment factors and patient factors as discussed below. It should be noted that percentages are overlapped as participants were not limited to one factor, so there is a possibility for multiple reasons for ART non adherence.

\subsubsection{Social factors}

Social factors were reported by $40(18.2 \%)$ as the causative factor for non-adherence to ART treatment. Among those participants, 2 (3.0\%) did not want to be seen taking ART while 10 (15.2\%) indicated that they are non-adhering to ART treatment due to alcohol abuse which cause them to forget to take medication.

\subsubsection{Economic factors}

Out of 66 participants $40(60.6 \%)$ indicated that economic factors cause them to be non-adhering to ART treatment as $20(30.3 \%)$ cited long distance to the ART clinic and the need to reach there with the taxi and most of the time the taxi money is not available. Furthermore, lack of food as ART requires not to be taken on empty stomach was stated by 20 (30.3\%).

\subsubsection{Employment or workplace factors}

Only $3(4.5 \%)$ of the participants indicated that work related travelling cause them not to adhere to ART treatment as sometimes they are abroad when they were suppose to go for follow up.

\subsubsection{Treatment factors}

Non adherence to ART medication due to the treatment factors was reported by $7(10.6 \%)$ such as non-availability of ART medication as indicated by $3(4.5 \%)$ participants while ART medication side effect was stated by 4 (6.1) participants. 


\subsubsection{Patient factors}

Among the participants, $4(6.1 \%)$ indicated that they were not adhering to the ART treatment due to forgetfulness to take medication.

\section{Discussion}

The current study assessed the non-adherent to ART treatment among male patients at Katutura Health Center, Windhoek, Namibia. The current study revealed the non- adherence to ART treatment to be higher among male patients ages 25 to 34 years. The finding is almost supporting Watt et al. (2010) study which shows that patients aged between 19 to 30 years are more likely to non-adhere to ART treatment. However, study by Okoronkwo et al. (2013) indicated the higher number of ART non adherent patients to be aged between 20-29 years.

In this study, the majority of the male patients who defaulted ART are single. This study findings is supporting the study conducted in Nigeria by Okoronkwo et al., (2013) which found out that marital status (being single) is statically associate with non-adherence of ART treatment. Furthermore, Okoronkwo'set al. (2013) study shows that the majority of non adherent patients were single. In contrast, study conducted by Berhe et al., (2013) in Ethiopia, found out that the majority of non-adherent patients were married. This current study findings shows that single patients are defaulting possibly because lack of family support.

According to the current study findings the majority of participants non adhering to ART treatment was found to be with secondary educational level. These findings concur with a study conducted by Okoronkwo et al. (2013) in which secondary education was associated with non-adherence to ART treatment. This can be attributed to the fact that low educated people are likely to have relevant information by attending health related community meetings. Furthermore, the majority of participants who defaulted ART were unemployed. Similar findings were reported by Berhe et al., (2013) during the study in Ethiopia whereby the majority of ARTnon adherent patients were unemployed.

The study finding showed that some participants were non adherent to ART treatment because they did not want to be seen taking ART. Similar sentiment was reported by Bauleth et al., ( 2013 ) in Namibia were participants indicated missed doses to preventing seen taking ART medication because of fear of stigma.

Other participants indicated that they are non-adhering to ART treatment due to alcohol abuse which is similar to a study conducted by Kip and colleagues (2009) in Botswana which reported that alcohol abuse was among the reported factors contributed to ART non adherence. The use of alcohol when on treatment may affect the user negatively by either affecting the effectiveness of the ART drugs or forgetting to take ART medication.

Another concern raised by participants is the long distance to the ART clinics and the lack of transport money for medication refill or follow up as contributing to non-adherence. Similarly, studies conducted in Namibia, also cited the same factors (Bauleth et al., 2013; Tuhadeleni et al., 2016). Although ART service is decentralized in Namibia there is a possibility of patients travel long distances to avoid being seen in the ART clinic near their residential area for fear of stigma.

Furthermore, lack of food was stated by some participants as challenge. This finding is supporting the study by Tuhadeleni et al., (2016) were by participants indicated lack of food as hindering ART adherence. According to a study conducted in Uganda, patients fear taking treatment on empty stomach and they often opt to interrupt treatment rather than continuously taking medication without food (McKinney et al., 2014).According to the WHO (2006) report, not having food may cause patients to skip medication and this may induce sub-optimal adherence.

In the current study finding, few participants indicated that work related travelling cause them not to adhere to ART treatment as sometimes they find themselves abroad by the time they would suppose to go for follow up. Similar sentiment was cited in the study by Bauleth et al., (2013) where participants indicated work migration especially when working abroad affect ART adherence. Non adherence to ART medication due to the treatment factors such as non-availability of ART medication at the ART clinics was also revealed as hindering ART adherence. Similar sentiment was indicated in the study by Okoronkwo et al .,(2013 ) in Nigeria as ARV medication shortage contributed to poor adherence .In the current study ART medication side effect was stated by very few participants among factors contributing to non-adherence. Demeke and Chanie, (2014) conducted a similar study in Ethiopia where by participants also indicated medication side effect such as nausea and vomiting, skin rashes and peripheral neuropathy as a challenge leads to non-adherence. Furthermore, the study is supported by study findings from several authors in Africa (Okoronkwo et al., 2013;Weiser et al.,2010 and Shigdel et al., 2013). Patients who are not well informed about ART side effect found it hard to tolerate side effect which leads to non-adherence (Wakibi et al . 2011). Forgetfulness to take medication was also revealed as contributed to non-adherence. The study finding is consistent to the findings of Bauleth et al.,2013,Okoronkwo et al.,2013,Shigdel et al., 2014, and Wakibi et al., 2011. The study findings also revealed that some participants indicated that they were not informed about the importance of ART adherence. The findings concur with Bauleth's et al., (2013) study which found out that poor knowledge of ART treatment was the reason provided by participants cause them to stop ARV when they felt better.

\section{Conclusion}

The study findings concluded that non-adherence of ART treatment in Katutura Health Centre, Khomas region is associated with socio economic status such as non-availability of food ,long distance to the ART facility of treatment, and alcohol abuse. Furthermore, other factors such as lack of knowledge on the importance of ART adherence, fear of stigma, ART medication side effects, ART medication shortage and work migration also contribute to ART non adherence. Community education programs should be developed to promote ART adherence. In addition, the MoHSS should ensure that Antiretroviral (ARV) medications should be available and accessible on all public health facilities in Namibia, to prevent non adherence related to medication shortage.

\section{Acknowledgement}

We would like to thank the Ministry of Health and Social Services research ethical committee for approving this study and providing the ethical clearance. We also thank the registered nurse in charge of Katutura Health Center for granting us permission to conduct this study .Lastly; our sincere gratitude goes to the participants who participated to this study voluntarily.

\section{References}

[1] Bauleth MF, Van Wyk B \&Ashipala DO (2016) Factors associated with poor adherence amongst patients receiving antiretroviral therapy at the Oshakati intermediate hospital in Namibia. International $\begin{array}{llll}\text { Journal of } & \text { Healthcare. } & 2 & (2):\end{array}$ 100.https://doi.org/10.5430/ijh.v2n2p89.

[2] Berhe N, Tegabu D, \&Alemayehu M (2013) Effect of nutritional factors on adherence to antiretroviral therapy among HIV-infected adults in North Ethiopia. BMC.Infectious Diseases. 13 (233):1-9.

[3] Demeke B \&Chanie T (2014) Adherence to antiretroviral therapy and associated factors among patients living with HIV/AIDS in Dessie Referral Hospital, Northern Ethiopia. International Journal of Pharma Sciences and Research.5: 572-581.

[4] Kip E, Ehlers VJ, van der Wal DM (2009) Patient's Adherence to Anti- Retroviral Therapy in Botswana. Journal of Nursing Scholar-

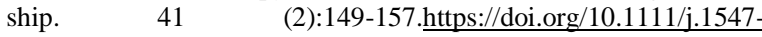
5069.2009.01266.x. 
[5] Makua T (2015) Reduced adherence to antiretroviral therapy in Polokwane, Limpopo province, South Africa. African Journal for Physical, Health Education, Recreation and Dance. (1): 107-114.

[6] McKinney O, Modeste NN, Lee J W, Gleason PC and MaynardTucker (2014) Determinants of Antiretroviral Therapy Adherence among Women in Southern Malawi: Healthcare Providers Perspectives. AIDS Research and Treatment: 19.https://doi.org/10.1155/2014/489370.

[7] Ministry of Health Social and Services (2014).Surveillance Report of the 2014 National HIV Sentinel Survey. Windhoek, Namibia.

[8] Ministry of Health Social and Services. Katutura Health Center January -June 2016 statistic report, 2016. Windhoek, Namibia.

[9] Ministry of Health and Social Services. (2013)Annual Report for 2012-2013. Windhoek, Namibia.

[10] Okoronkwo I, Okeke U, Chinweuba A, and Iheanacho P ( 2013) Nonadherence Factors and Socio- demographic Characteristics of HIV-Infected Adults Receiving Antiretroviral Therapy in NnamdiAzikiwe University Teaching Hospital, Nnewi, Nigeria . ISRN AIDS: 2-9.

[11] Reda A and Biadgilign S (2012) Determinants of Adherence to Antiretroviral Therapy amongHIV-Infected Patients in Africa. AIDS Research and Treatment: 1-8.https://doi.org/10.1155/2012/574656.

[12] ShigdelR,KloumanE,Bhandari A and Ahmed L A (2014) Factors associated with adherence to antiretroviral therapy in HIV-infected patients in Kathmandu District, Nepal. HIV/AIDS - Research and Palliative Care.6: 109-116.https://doi.org/10.2147/HIV.S55816.

[13] Tjituka F, Gweshe J, Mabirizi D, Sumbi V, Lukwago J \&Sagwa E (2013) Namibia Antiretroviral Therapy Adherence Baseline Survey Report. Windhoek. Namibia.

[14] Tuhadeleni O, Gary E, Ashipala DO and Nuuyoma V (2016) the Perceptions of HIV-Positive Patients (ART Patients) on AntiRetroviral Therapy (ART), Treatment Supporters and Health Care Workers with Regard to their Role in ART Adherence at ART Clinics in the Intermediate Hospital Oshakati, Namibia. Health Science Journal. 5 (10):1-7.

[15] Science Journal

[16] UNAIDS (2006) Report on global AIDS epidemic. Geneva: Join United Nations Programme on AIDS.

[17] Wakibi SN, Ng'ang'a ZW and Mbugua GG (2011) Factors associated with non-adherence to highly active antiretroviral therapy in Nairobi, Kenya. AIDS Research and Therapy. 8 (43): 18.https://doi.org/10.1186/1742-6405-8-43.

[18] Watt MH, Maman S, Golin CE, Earp JA, Eng E, Bangdiwala S \& Jacobson M (2010) Factors associated with self-reported adherence to antiretroviral therapy in a Tanzanian setting. AIDS Care. 22(3): 381-389.https://doi.org/10.1080/09540120903193708.

[19] Weiser SD, Tuller DM, Frongillo EA, Senkungu J, Mukiibi N, Bangsberg DR: Food insecurity as a barrier to sustained antiretroviral therapy adherence in Uganda. PLoS One 2010, 5(4). https://doi.org/10.1371/journal.pone.0010340.

[20] World Health Organization (2006).Antiretroviral Therapy for HIV infection in adults and Adolescents in Resource-Limited settings: Towards Universal Access. Geneva: Switzerland. 